\title{
Safety and Efficacy of Nitazoxanide-Based Regimen for the Eradication of Helicobacter pylori Infection: A Systematic Review and Meta-Analysis
}

\author{
Umair Iqbala, g, Harshit S. Khara ${ }^{\mathrm{a}}$, Daud Akhtarb, Yirui Huc ${ }^{\mathrm{c}}$, Hafsa Anwar ${ }^{\mathrm{d}}$, Khwaja F. Haq ${ }^{\mathrm{e}}$, \\ Hafiz U. Siddiquif ${ }^{f}$, Marika K. Bergenstock ${ }^{\mathrm{a}}$, Matthew J. Shellenberger ${ }^{\mathrm{a}}$
}

\begin{abstract}
Background: Helicobacter pylori (HP) is the most common cause of gastritis worldwide. Clarithromycin-based triple therapy or bismuthbased quadruple therapy is usually considered the first-line treatment, however with around 30\% failure rate for both regimens. Drug resistance of clarithromycin and metronidazole is a growing concern in some parts of the world. Therefore, there is a need for effective eradication regimen for HP. Nitazoxanide, a bactericidal thiazolide antibiotic, has been shown to be effective in HP infection. We conducted a systematic review and meta-analysis to evaluate the efficacy of nitazoxanide-based regimen for the eradication of HP.
\end{abstract}

Methods: We have searched PubMed, Embase, Ovid Medline and Cochrane library database from inception to December 9, 2020 to identify studies that utilized nitazoxanide in the treatment regimen for HP eradication. Our primary outcome was pooled eradication rate of HP.

Results: Thirteen studies including 1,028 patients met our inclusion criteria and were analyzed in a meta-analysis. HP eradication was successful in 867 patients with a pooled eradication rate of $86 \%$ (95\% confidence interval (CI): $79-90 \%$ ) with $84 \%$ heterogeneity. A subgroup analysis that included 230 patients who failed other prior

Manuscript submitted November 17, 2020, accepted December 16, 2020

Published online December 23, 2020

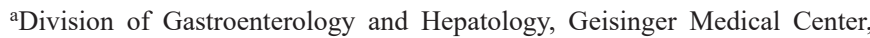
Danville, PA 17822, USA

${ }^{b}$ Department of Medicine, University of British Columbia, Vancouver, BC V6T 1Z4, Canada

'Department of Population Health Sciences, Geisinger Medical Center, Danville, PA 17822, USA

dDepartment of Internal Medicine, Capital Health Regional Medical Center, Trenton, NJ 08638, USA

eDivision of Gastroenterology and Hepatology, Henry Ford Hospital, 2799 W Grand Blvd, Detroit, MI 48202, USA

fDepartment of Surgery, Cleveland Clinic Foundation, 9500 Euclid Ave, Cleveland, OH 44195, USA

'Corresponding Author: Umair Iqbal, Department of Gastroenterology and Hepatology, Geisinger Medical Center, Danville, PA 17822, USA.

Email: umairiqbal_dmc@hotmail.com

doi: https://doi.org/10.14740/gr1342 eradication regimens revealed a pooled eradication rate of $85 \%(95 \%$ CI: $69-94 \%)$ without heterogeneity. In a subgroup analysis, highest eradication rates were achieved with levofloxacin, doxycycline, nitazoxanide and proton pump inhibitor with a pooled eradication rate of $92 \%(88-95 \%)$.

Conclusion: Nitazoxanide-based regimen is safe and effective in the eradication of HP infection. It is also successful as a salvage therapy in patients who have failed prior treatments.

Keywords: Nitazoxanide; Helicobacter pylori; Eradication regimen; Meta-analysis

\section{Introduction}

Helicobacter pylori (HP), a gram-negative bacillus, is a potent bacterial colonizer of human gastric mucosa with an estimated $50 \%$ of the world's population chronically infected [1]. Chronic infection due to HP can subsequently lead to the development of certain gastrointestinal (GI) complications, such as chronic gastritis, peptic ulcer disease and gastric malignancies such as gastric adenocarcinoma and mucosal-associated lymphoid tissue (MALT) lymphomas. The eradication of HP plays a crucial role in reducing the incidence of these complications [2-4]. The increasing prevalence of antibiotic resistance has decreased eradication rates (ERs) with current first-line guideline recommended strategies that include standard triple therapy (proton pump inhibitor (PPI), clarithromycin, and amoxicillin or metronidazole) or bismuth-based quadruple therapy (PPI or $\mathrm{H}_{2}$ receptor antagonists, bismuth, metronidazole and tetracycline) [5-7]. More recently, sequential therapy of these treatment options has also been introduced [8]. Specifically, recent studies have reported resistant rates to clarithromycin ranging from $18 \%$ in Europe to as high as $50 \%$ in Asia. Resistance to metronidazole, similarly, has been reported to range between $30 \%$ and $40 \%$ in developed and as high as $80 \%$ in developing nations across the globe $[5,7,9]$. Such overwhelming antibiotic resistance has warranted a critical need for alternative therapeutic options.

Over the last decade, nitazoxanide (NTZ), a broad-spectrum thiazolide antibiotic that is characteristically similar to 
metronidazole, has emerged as a potential treatment of HP [10]. Presently, NTZ, a compound with anti-bacterial, anti-viral and anti-parasitic activity, is the first-line therapy for treatment of Cryptosporidium parvum and Giardia lamblia [11]. Recently, studies have shown effective eradication of HP with treatments including NTZ. Here, we aimed to evaluate the efficacy and safety of treatment strategies that incorporate NTZ for HP infection treatment.

\section{Materials and Methods}

\section{Search strategy}

We have searched PubMed, Embase, Ovid Medline and Cochrane library database from inception to December 9, 2020 to identify studies which utilized NTZ in the treatment regimen for HP eradication. A manual search was also performed through the bibliographies of the retrieved publications to increase the yield of possibly relevant studies. Two investigators independently conducted the systematic literature review. Observational studies or randomized controlled trials (RCTs) that evaluated NTZ as part of eradication regimen for HP were eligible for inclusion in the meta-analysis. The sample size of a study did not restrict its inclusion in the meta-analysis. When possible, we only included patients who completed the full length of therapy and were tested for eradication on follow-up. Our primary outcome was pooled ER of HP. We also performed a subgroup analysis to evaluate ER of NTZ-based regimen in patients who failed prior treatment. This study was conducted in agreement with the Preferred Reporting Items for Systematic Reviews and Meta-Analysis statement [12]. Quality assessment of the observational studies was performed by using Newcastle-Ottawa quality assessment scale and Jadad score was utilized to assess the quality of RCT. Quality assessment did not affect the eligibility of the studies in the meta-analysis. Institutional review board approval and ethical compliance were not needed given this is a meta-analysis.

\section{Data extraction}

A structured data collection form was used to extract the following data from each study: the title of the study, publication year, name of the first author, country where the study was performed, number of participants, characteristics of the participants, eradication regimen, ER, dose of NTZ used and adverse events. To ensure accuracy, data extraction was independently performed by two investigators (UI and KFH) and was reviewed by the third investigator (HUS). Any disagreement was resolved by mutual agreement.

\section{Statistical analysis}

Incidence rates (IRs) and confidence intervals (CIs) were initially calculated for the primary outcome (ER) from included studies. The pooled IR is considered statistically significant if 95\% CI did not contain 1. Forest plots were then made using pooled estimates and measure of variability of each included study. Publication bias was evaluated by funnel plot diagram. Variability between included studies was assessed by heterogeneity tests using $\mathrm{I}^{2}$ statistic. All analyses were conducted using RStudio (Version 1.0.136; Boston, MA) using the "Meta" and "Metafor" package.

\section{Results}

Initial search revealed 172 articles. After removal of duplicates, editorials and case reports 20 articles underwent full review. Out of 20 articles, 13 studies including 1,028 patients met our inclusion criteria and were analyzed in a meta-analysis as summarized in Table 1 [13-25]. Figure 1 elaborates the systematic review process of our meta-analysis. There were eight observational studies and five randomized clinical trials. All the randomized trials are fair quality. Among observational studies, seven were of good quality and one was of fair quality. Table 1 elaborates complete eradication regimen, dosage of NTZ and patient population included in each study.

Out of 1,028 patients, HP eradication was successful in 867 patients with a pooled ER of $86 \%(95 \%$ CI: $79-90 \%)$ with $84 \%$ heterogeneity (Fig. 2). We also performed a subgroup analysis including 230 patients who have failed other prior eradication regimens and revealed a pooled ER of $85 \%(95 \%$ CI: $69-94 \%$ ) without heterogeneity. No serious adverse events were reported in the studies. Table 1 reports minor adverse events reported in each study. In a subgroup analysis, highest ERs were achieved with levofloxacin, doxycycline, NTZ and PPI with a pooled ER of $92 \%$ (88-95\%) (Fig. 3). There was no publication bias as evaluated by funnel plot diagram (Fig. 4).

\section{Discussion}

The increasing resistance to antibiotics poses an important challenge with regards to the treatment of HP. Specifically, a previous meta-analysis of over 53,000 patients demonstrated a cure rate of less than $80 \%$ for guideline recommended first-line therapy $[26,27]$. Furthermore, the number of patients who are treatment refractory continues to rise, with evidence suggesting that at least $10 \%$ of patients infected with HP will fail both first- and second-line regimens [28]. For these reasons, there is a growing need to find other suitable treatment modalities such as those that include NTZ.

In this systematic review and meta-analysis, we demonstrated that HP eradication with regimens containing NTZ is an effective and safe strategy. HP pooled ERs with NTZ regimens equated to $83 \%(95 \% \mathrm{CI}: 76-90 \%)$ without heterogeneity (P $=0.17)$. Furthermore, subgroup analysis of 230 patients with failed eradication of HP revealed a pooled ER of $80 \%(95 \%$ CI: 66-94\%) without heterogeneity $(\mathrm{P}=0.39)$. Treatment regimens that included levofloxacin, doxycycline, NTZ and PPIs were the most effective, achieving the highest HP pooled ER of $92 \%(82-100 \%)$. With regards to safety, no serious adverse 


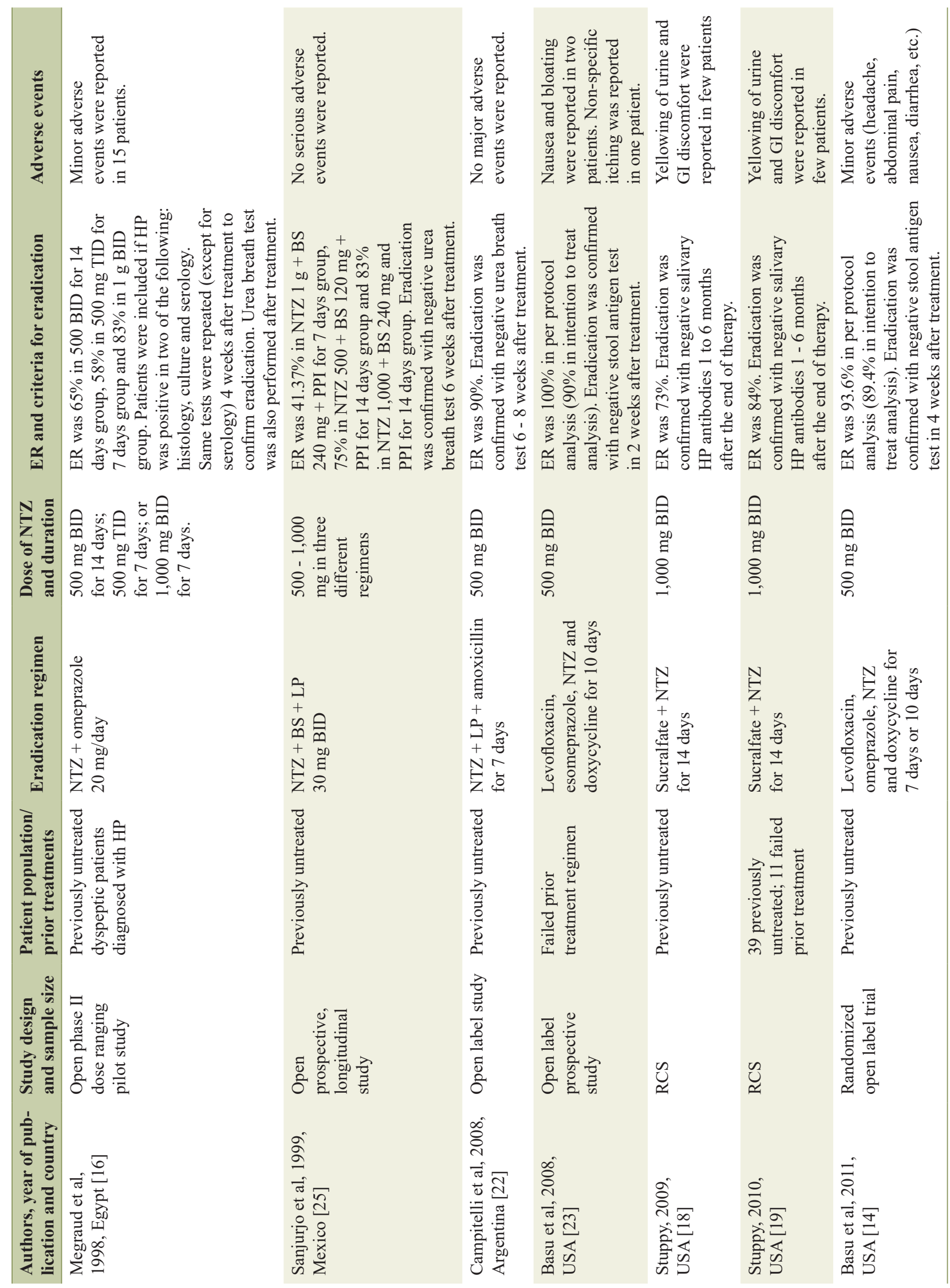




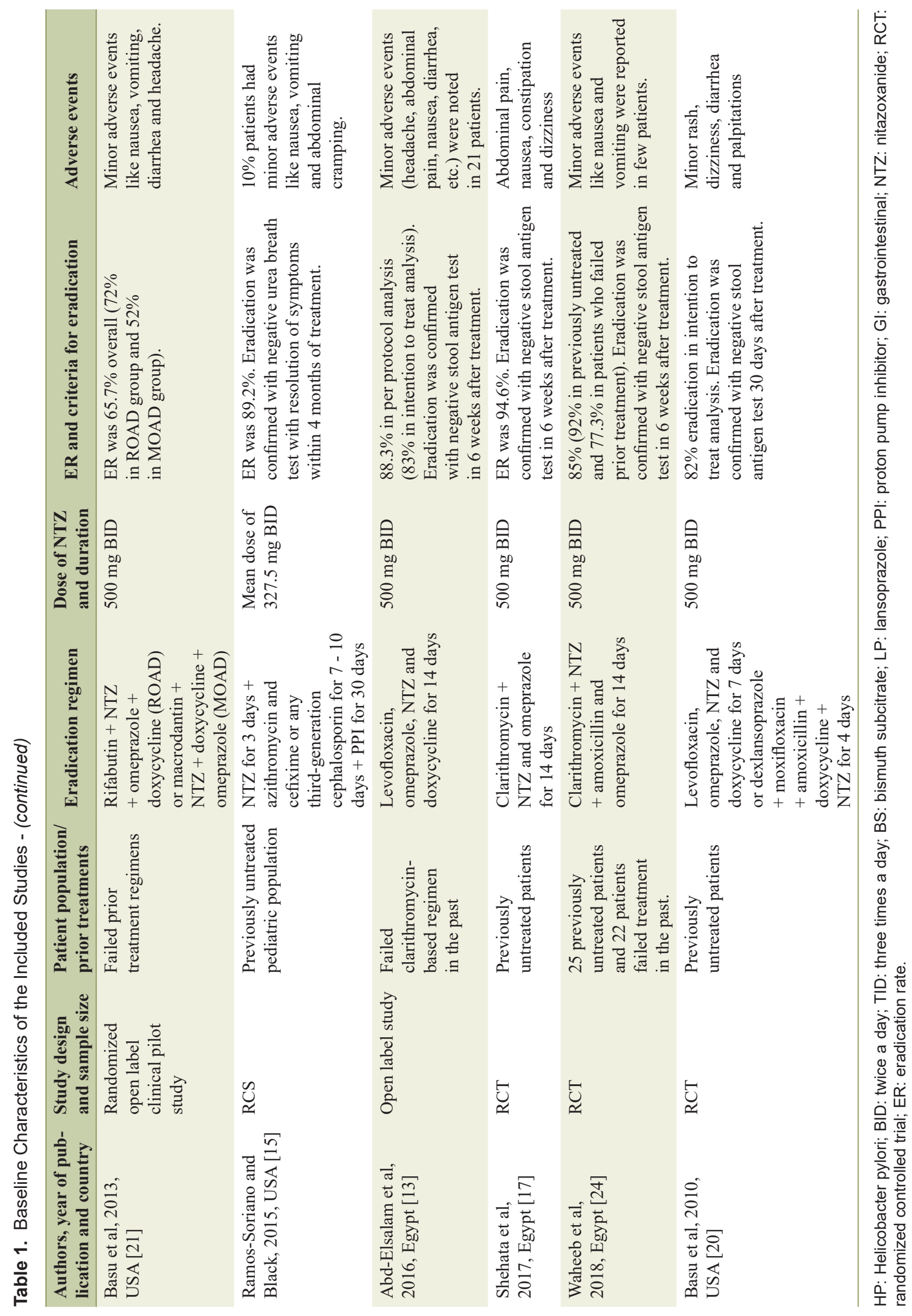




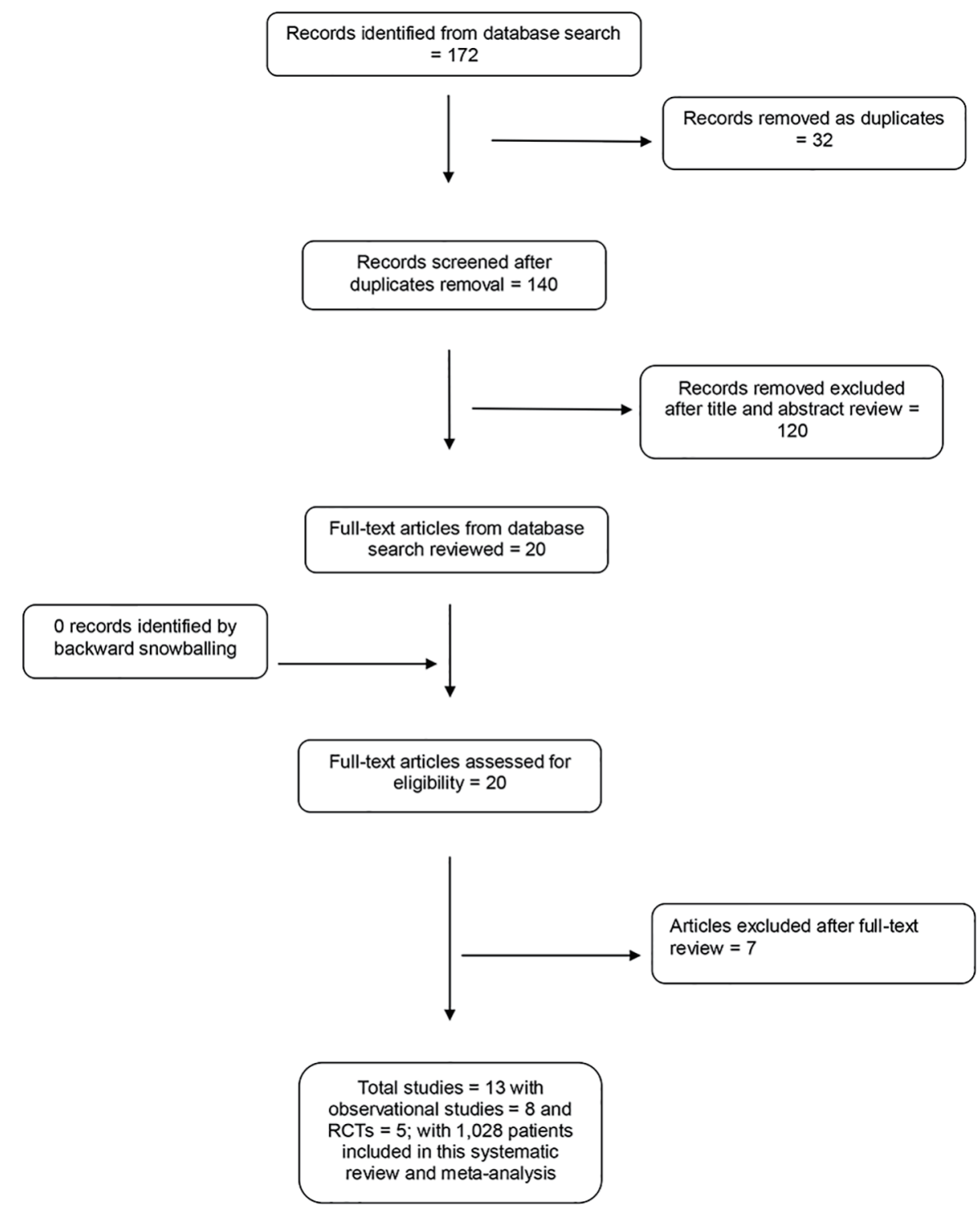

Figure 1. PRISMA flow diagram.

events were reported.

NTZ is a thiazolide antibiotic that exerts its bactericidal activity via mechanisms that prevent anaerobic energy metabolism via the inhibition of pyruvate oxidoreductase enzyme [29]. Although characteristically similar to metronidazole, NTZ differs due to its anti-vacuolating toxin activity that prevents against the formation of antimicrobial resistance. These findings are supported by in vitro studies that demonstrate NTZ to be a potent agent against metronidazole-resistant strains [16, 30]. These characteristics of NTZ may explain the success of NTZ containing regimens as both first-line and against multidrug-resistant HP strains. The success rates of NTZ regimens are as high as $80 \%$, as shown in this meta-analysis, against multidrug-resistant HP strains, and may point to a synergistic interaction between NTZ and other antibiotics.

Treatment regimens that included levofloxacin, doxycycline, NTZ and PPIs achieved an ER of 92\%, which achieves a grade B or good rating as per Graham's reporting scale [31]. Such successful ERs may be due to the activity of levofloxacin on DNA gyrase, allowing it to interfere with bacterial DNA replication [32]. Another important factor is the use of doxycycline, a tetracycline analog. Doxycycline inhibits bacterial protein synthesis in order to exert its bacteriostatic effects. Studies have shown little or no resistance to doxycycline and support the use of doxycycline as a rescue therapy in the treatment of HP [33-35].

Presently, PPIs form the backbone of any treatment regimen against HP. PPIs alter gastric acidity, thereby disrupting the optimal environment for HP replication. Omeprazole and esomeprazole were the primary PPIs utilized in the studies included in this meta-analysis.

In terms of safety, clinical studies with NTZ have shown successful use with no significant adverse events at total daily doses ranging from 1,000 to $2,000 \mathrm{mg}$ as well as $500 \mathrm{mg}$ 


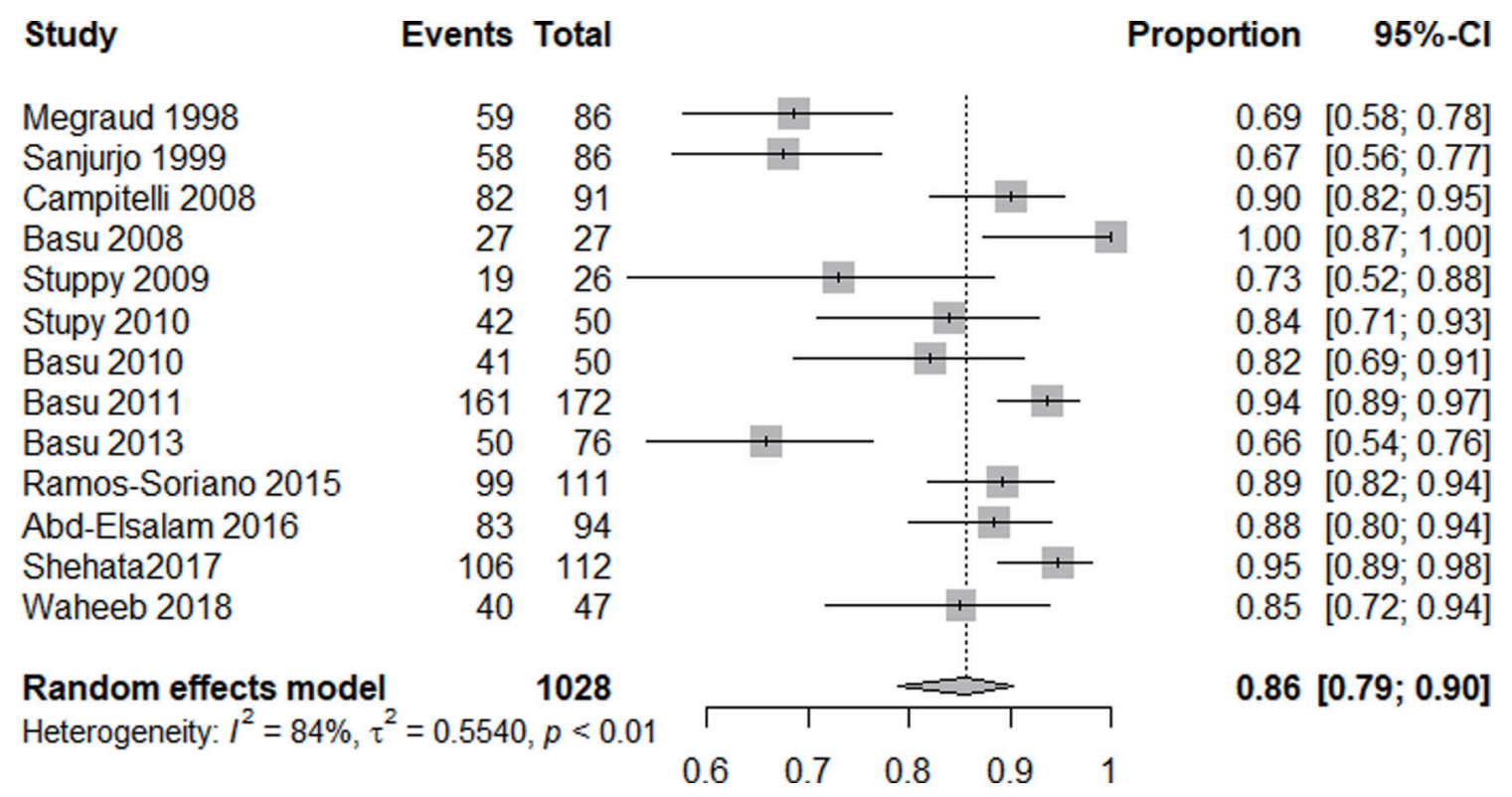

Figure 2. Pooled eradication rate.

when used in combination with other antibiotics [5, 14, 19]. Our meta-analysis supported these findings with no reported serious adverse events occurring in the included studies. Minor adverse events such as abdominal pain, headache, nausea, change in urinary color and diarrhea were reported in these studies. These findings suggest that NTZ containing therapies are a safe alternative and that lower doses of $500 \mathrm{mg}$ might suffice in the treatment of HP.

The systematic review process for the meta-analysis was comprehensive and we included all available studies to date. We recognize, however, that there are certain limitations of this meta-analysis, and thus, the results should be interpreted with caution. Considerations that may have influenced the pooled ERs include the duration of treatment, which varied from 4 to 14 days, dosage variability as well as the region in which the studies were performed. Our meta-analysis included studies from North America, South America and Africa with no studies from Asia. HP infection is a global health dilemma with varying patterns depending on geographic location, and thus our results may not represent findings in places such as Asia.

In conclusion, this meta-analysis indicates that NTZ- based regimens are safe and effective in the eradication of HP infection. Additionally, these regimens can also play a role as rescue therapies in patients with multidrug-resistant infections who have failed first- or second-line treatments. The specific combination of levofloxacin, doxycycline, NTZ and PPIs may be a reasonable therapeutic option based on antimicrobial susceptibility testing. Although these findings are promising, further research in the form of larger randomized controlled studies are required to delineate the most effective NTZ-based regimens for the eradication of $\mathrm{HP}$.

\section{Acknowledgments}

We would like to thank Stephanie Stebens from Sladen Library, Henry Ford Hospital (Detroit, MI, USA) for her contribution in formatting and proofreading the manuscript. We also would like to thank Gina Hug for assisting us in systematic search. We presented this study as a poster presentation at The American College of Gastroenterology Annual Meeting 2019.

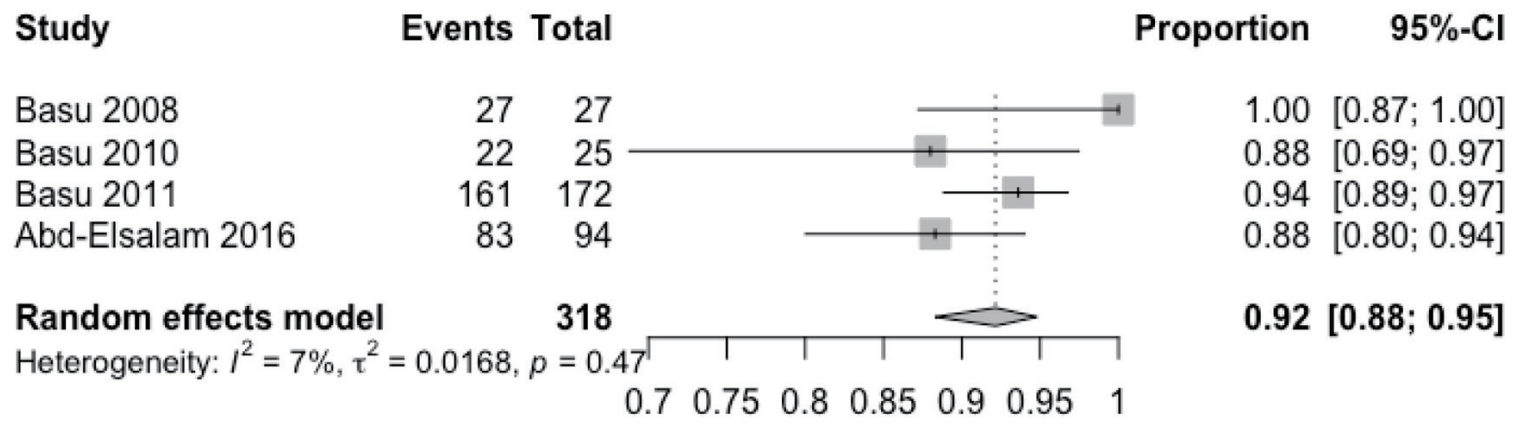

Figure 3. Pooled eradication rate of levoflaxacin, doxycyline, nitazoxanide and proton pump inhibitor therapy. 


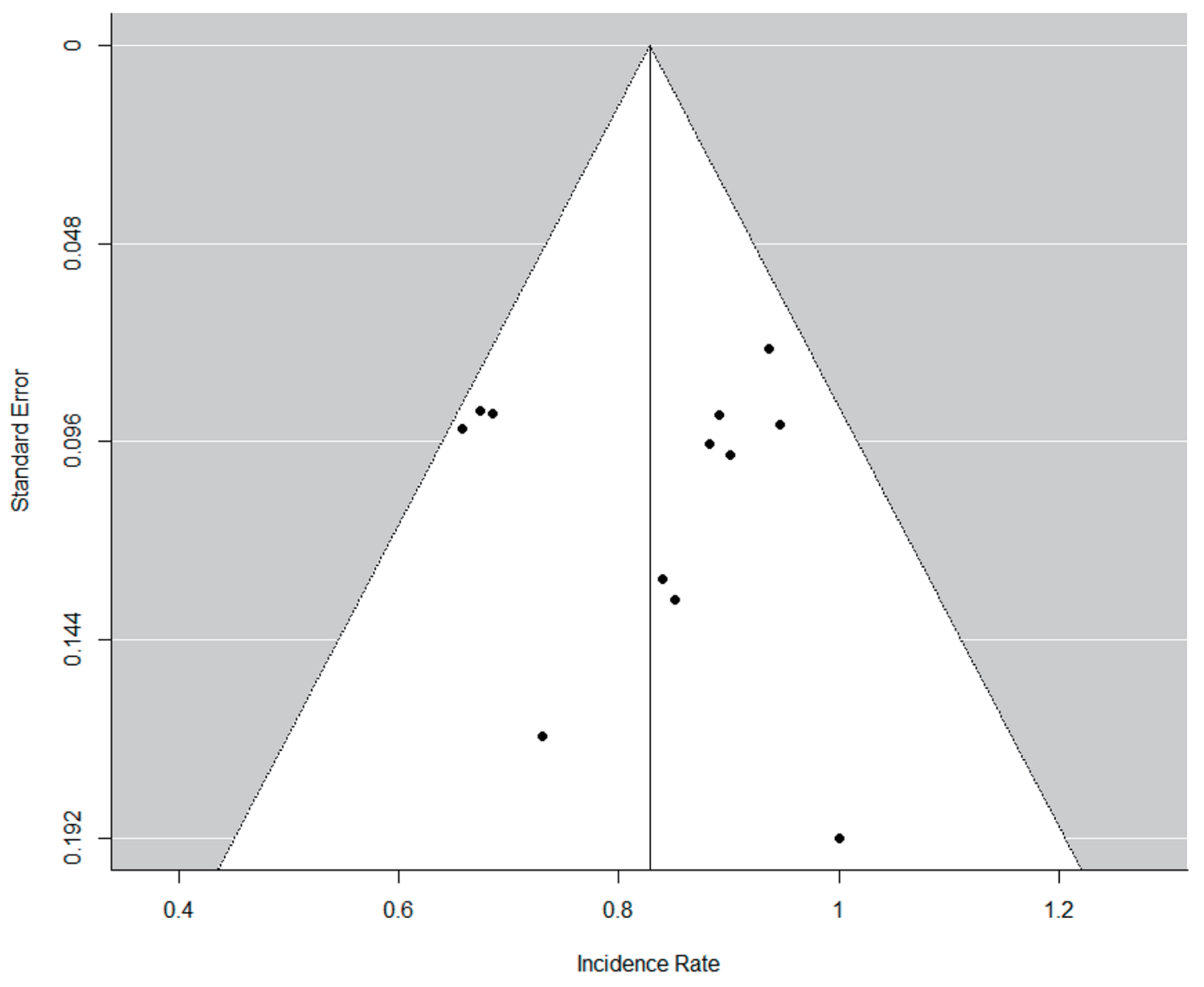

Figure 4. Funnel plot for publication bias.

\section{Financial Disclosure}

None to declare.

\section{Conflict of Interest}

None to declare.

\section{Informed Consent}

Not applicable.

\section{Author Contributions}

Umair Iqbal: data abstraction, writing manuscript, quality assessment and statistical analysis. Harshit S. Khara: manuscript writing and supervising the research project. Yirui Hu: synthesis of results and statistical analysis. Hafiz Umair Siddiqui: literature search and manuscript writing. Hafsa Anwar: literature search and manuscript writing. Khwaja F. Haq: search strategy. Marika Bergenstock: literature search and quality assessment. Daud Akhtar: manuscript writing. Matthew J. Shellenberger: reviewing and supervising the project.

\section{Data Availability}

The authors declare that data supporting the findings of this study are available within the article.

\section{References}

1. Hunt RH, Xiao SD, Megraud F, Leon-Barua R, Bazzoli F, van der Merwe S, Vaz Coelho LG, et al. Helicobacter pylori in developing countries. World Gastroenterology Organisation Global Guideline. J Gastrointestin Liver Dis. 2011;20(3):299-304.

2. McColl KE. Clinical practice. Helicobacter pylori infection. N Engl J Med. 2010;362(17):1597-1604.

3. Malfertheiner P, Megraud F, O'Morain CA, Gisbert JP, Kuipers EJ, Axon AT, Bazzoli F, et al. Management of 
Helicobacter pylori infection-the Maastricht V/Florence Consensus Report. Gut. 2017;66(1):6-30.

4. Ford AC, Forman D, Hunt RH, Yuan Y, Moayyedi P. Helicobacter pylori eradication therapy to prevent gastric cancer in healthy asymptomatic infected individuals: systematic review and meta-analysis of randomised controlled trials. BMJ. 2014;348:g3174.

5. Megraud F, Coenen S, Versporten A, Kist M, Lopez-Brea M, Hirschl AM, Andersen LP, et al. Helicobacter pylori resistance to antibiotics in Europe and its relationship to antibiotic consumption. Gut. 2013;62(1):34-42.

6. Kuo YT, Liou JM, El-Omar EM, Wu JY, Leow AHR, Goh KL, Das R, et al. Primary antibiotic resistance in Helicobacter pylori in the Asia-Pacific region: a systematic review and meta-analysis. Lancet Gastroenterol Hepatol. 2017;2(10):707-715.

7. Thung I, Aramin H, Vavinskaya V, Gupta S, Park JY, Crowe SE, Valasek MA. Review article: the global emergence of Helicobacter pylori antibiotic resistance. Aliment Pharmacol Ther. 2016;43(4):514-533.

8. Xin Y, Manson J, Govan L, Harbour R, Bennison J, Watson $\mathrm{E}, \mathrm{Wu} \mathrm{O}$. Pharmacological regimens for eradication of Helicobacter pylori: an overview of systematic reviews and network meta-analysis. BMC Gastroenterol. 2016;16(1):80.

9. Horiki N, Omata F, Uemura M, Suzuki S, Ishii N, Iizuka Y, Fukuda K, et al. Annual change of primary resistance to clarithromycin among Helicobacter pylori isolates from 1996 through 2008 in Japan. Helicobacter. 2009;14(5):86-90.

10. Guttner Y, Windsor HM, Viiala CH, Dusci L, Marshall BJ. Nitazoxanide in treatment of Helicobacter pylori: a clinical and in vitro study. Antimicrob Agents Chemother. 2003;47(12):3780-3783.

11. Anderson VR, Curran MP. Nitazoxanide: a review of its use in the treatment of gastrointestinal infections. Drugs. 2007;67(13):1947-1967.

12. Moher D, Liberati A, Tetzlaff J, Altman DG, the PRISMA Group. Preferred reporting items for systematic reviews and meta-analyses: the PRISMA statement. Ann Intern Med. 2009;151(4):264-269, W264.

13. Abd-Elsalam S, Kobtan A, El-Kalla F, Elkhalawany W, Nawasany SE, Saif SA, Yousef M, et al. A 2-week Nitazoxanide-based quadruple treatment as a rescue therapy for Helicobacter pylori eradication: A single center experience. Medicine (Baltimore). 2016;95(24):e3879.

14. Basu PP, Rayapudi K, Pacana T, Shah NJ, Krishnaswamy N, Flynn M. A randomized study comparing levofloxacin, omeprazole, nitazoxanide, and doxycycline versus triple therapy for the eradication of Helicobacter pylori. Am J Gastroenterol. 2011;106(11):1970-1975.

15. Ramos-Soriano AG, Black J. Nitazoxanide use as part of an empiric multi-drug regimen in treating children with suspected helicobacter pylori infection. Case Rep Gastroenterol. 2015;9(1):36-42.

16. Megraud F, Occhialini A, Rossignol JF. Nitazoxanide, a potential drug for eradication of Helicobacter pylori with no cross-resistance to metronidazole. Antimicrob Agents Chemother. 1998;42(11):2836-2840.
17. Shehata MA, Talaat R, Soliman S, Elmesseri H, Soliman S, Abd-Elsalam S. Randomized controlled study of a novel triple nitazoxanide (NTZ)-containing therapeutic regimen versus the traditional regimen for eradication of Helicobacter pylori infection. Helicobacter. 2017;22(5).

18. Stuppy W. Nitazoxanide and sucralfate for the treatment of helicobacter pylori infection: 139. Am J Gastroenterol. 2009;104:S55-S56.

19. Stuppy W. Dual therapy: nitazoxanide and sucralfate for the treatment of helicobacter pylori: 121. Am J Gastroenterol. 2010;105:S45-S46.

20. Basu PP, Krishnaswamy N, Korapati R, et al. A new four-day regimen with dexlansoprazole, moxifloxacin, amoxicillin, nitazoxanide, and doxycycline (DeMAND) in helicobacter pylori therapy: an open-label randomized clinical trial: 216. Am J Gastroenterol. 2010;105:S81.

21. Basu P, Shah NJ, Mittimani K, et al. Sa1928 Rifabutin, Omeprazole, Alinia and Doxycycline Therapy for prior treatment failure helicobacter pylori (HP) population - a randomized open label clinical pilot study - road trial. Gastroenterology. 2013;144(5):S-336.

22. Campitelli E, Paszkiewich A, Ibarra D, et al. Efficacy of a nitazoxanide based regimen for heliobacter pylori (HP) eradication: 109. Am J Gastroenterol. 2008;103:S43-S44.

23. Basu PP, Rayapudi K, Esteves J. LEND (L Evofloxacin, E Someprazole, N Itazoxanide and D Oxycycline) for the treatment of previously non-responsive heliobacter pylori: 133. Am J Gastroenterol. 2008;103:S52-S53.

24. Waheeb AA, Dorrah AO, Mousa WM, et al. A two-week nitazoxanide-based quadruple treatment as a rescue therapy for helicobacter pylori eradication. Egyptian Journal of Hospital Medicine. 2018;72(5):4509-4514.

25. Sanjurjo GJL, Perez MJ, Hidalgo LH, et al. Efficacy and safety amongst different doses of nitazoxanide + bismut subcitrate + lansoprazol to eradicate Helicobacter pylori. Rev Med Hosp Gen Mex. 1999;62(3):172-175.

26. Papastergiou V, Georgopoulos SD, Karatapanis S. Treatment of Helicobacter pylori infection: Past, present and future. World J Gastrointest Pathophysiol. 2014;5(4):392399.

27. Gisbert JP, Pajares JM. Treatment of Helicobacter pylori infection: the past and the future. Eur J Intern Med. 2010;21(5):357-359.

28. Rokkas T, Sechopoulos P, Robotis I, Margantinis G, Pistiolas D. Cumulative H. pylori eradication rates in clinical practice by adopting first and second-line regimens proposed by the Maastricht III consensus and a third-line empirical regimen. Am J Gastroenterol. 2009;104(1):2125.

29. Sisson G, Goodwin A, Raudonikiene A, Hughes NJ, Mukhopadhyay AK, Berg DE, Hoffman PS. Enzymes associated with reductive activation and action of nitazoxanide, nitrofurans, and metronidazole in Helicobacter pylori. Antimicrob Agents Chemother. 2002;46(7):21162123.

30. Yamamoto Y, Hakki A, Friedman H, Okubo S, Shimamura T, Hoffman PS, Rossignol J. Nitazoxanide, a nitrothiazolide antiparasitic drug, is an anti-Helicobacter pylori agent with anti-vacuolating toxin activity. Chemotherapy. 
1999;45(4):303-312.

31. Graham DY, Lu H, Yamaoka Y. A report card to grade Helicobacter pylori therapy. Helicobacter. 2007;12(4):275278.

32. Lee JW, Kim N, Nam RH, Park JH, Kim JM, Jung HC, Song IS. Mutations of Helicobacter pylori associated with fluoroquinolone resistance in Korea. Helicobacter. 2011;16(4):301-310.

33. Smilack JD. The tetracyclines. Mayo Clin Proc. 1999;74(7):727-729.

34. Cammarota G, Martino A, Pirozzi G, Cianci R, Branca
G, Nista EC, Cazzato A, et al. High efficacy of 1-week doxycycline- and amoxicillin-based quadruple regimen in a culture-guided, third-line treatment approach for Helicobacter pylori infection. Aliment Pharmacol Ther. 2004;19(7):789-795.

35. Taghavi SA, Jafari A, Eshraghian A. Efficacy of a new therapeutic regimen versus two routinely prescribed treatments for eradication of Helicobacter pylori: a randomized, double-blind study of doxycycline, co-amoxiclav, and omeprazole in Iranian patients. Dig Dis Sci. 2009;54(3):599-603. 